\title{
ПРИМЕНЕНИЕ ПРОЦЕССНОГО ПОДХОДА ДЛЯ КОМБИНИРОВАНИЯ ТЕХНОЛОГИЙ ОЦЕНКИ РИСКОВ
}

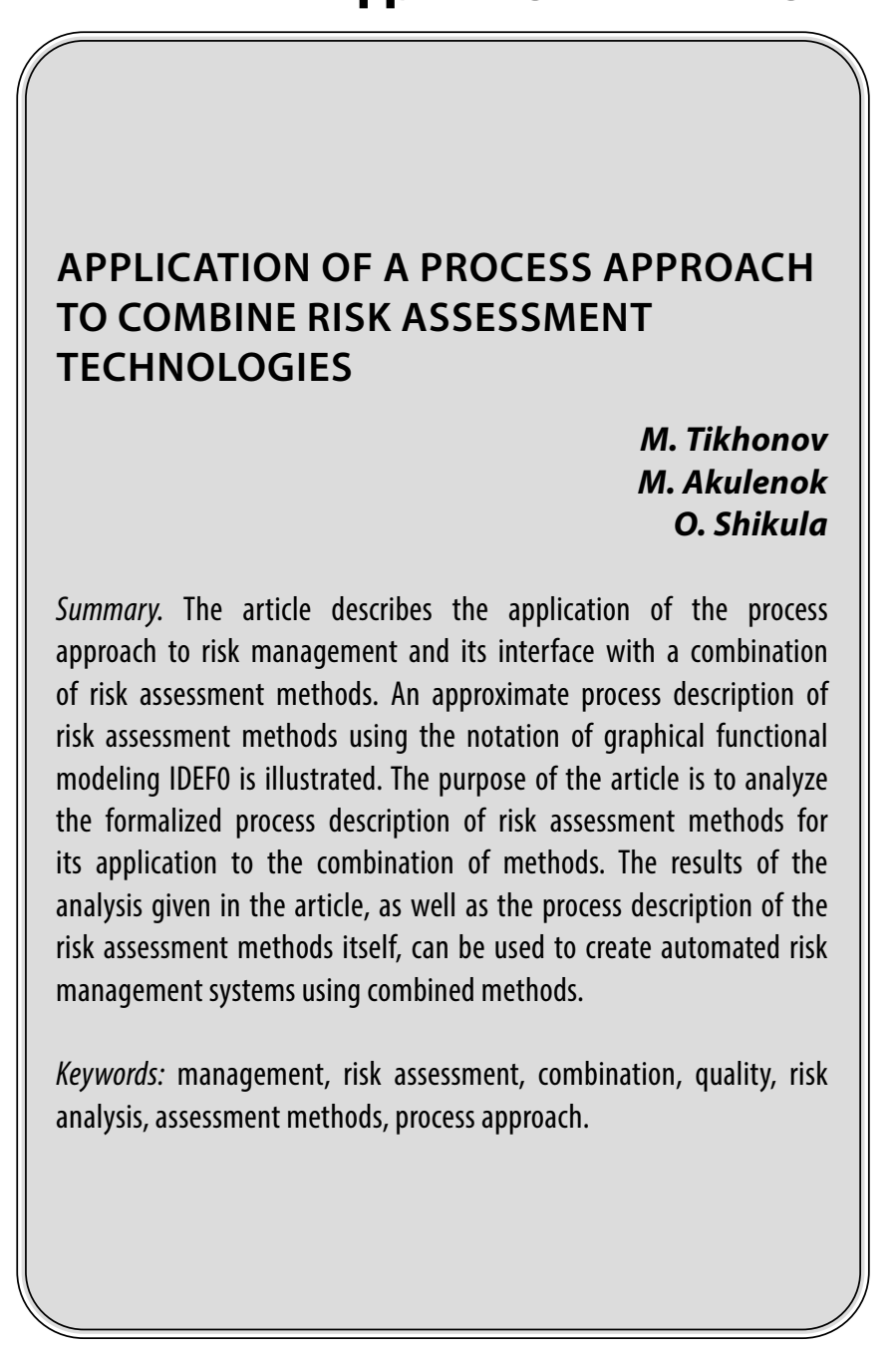

$\mathbf{0}$ ценка рисков является неотъемлемой составляющей управления процессами организацией и (или) проектами. Применение различных технологий такой оценки позволяет достичь результатов и выявить широкий спектр возможных рисков для любой деятельности. Однако разнообразие объектов оценки и особенности технологий оценки не позволяют провести исчерпывающий анализ рисков и верно установить текущую и предполагаемую ситуацию в организации с точки зрения её рисков и возможностей.

Трудоемкость процесса увеличивает применение оценки рисков в ручном режиме.

Для устранения перечисленных проблем целесообразно использовать комбинированные технологии
Тихонов Мартин Робертович

К.т.н., доцент, Национальный исследовательский университет «МИЭТ»

kurotenshi91@yandex.ru

Акуленок Марина Викторовна

К.т.н., дочент, Национальный исследовательский университет «МИЭТ»

amv@s2q.ru

Шикула Ольга Сергеевна

Старший преподаватель, Национальный исследовательский университет «МИЭТ»

oshik78@mail.ru

Аннотация. В статье приведен анализ применения процессного подхода к управлению рисками и его сопряжения с комбинированием технологий оценки рисков. Проиллюстрирован вариант процессного описания технологий оценки рисков с использованием нотации графического функционального моделирования IDEF0. Целью статьи является анализ формализованного процессного описания технологий оценки рисков на предмет его применения к комбинированию технологий. Результаты анализа, приведённые в статье, а также само процессное описание технологий оценки рисков могут быть использованы при создании автоматизированных систем управления рисками комбинированными технологиями.

Ключевые слова: управление, оценка рисков, комбинирование, качество, анализ рисков, методы оценки, процессный подход.

оценки рисков, автоматизировать частично (или полностью) процесс обработки данных. Повысить эффективность оценки, ее точность и получить разностороннюю оценку позволит сочетание этапов оценки различными технологиями при идентификации и при анализе рисков.

Для оптимального комбинирования применяемых технологий оценки рисков и преодоления возможных барьеров, потери данных применим процессный подход. В рамках данной работы под процессным подходом понимается определение границ действий технологий оценки рисков (выделение процессов), установление соответствующих атрибутов (например, их входов и выходов на всех этапов, а именно поступающие или полученные данные или информационные элементы) и определение путей их взаимодействия. 


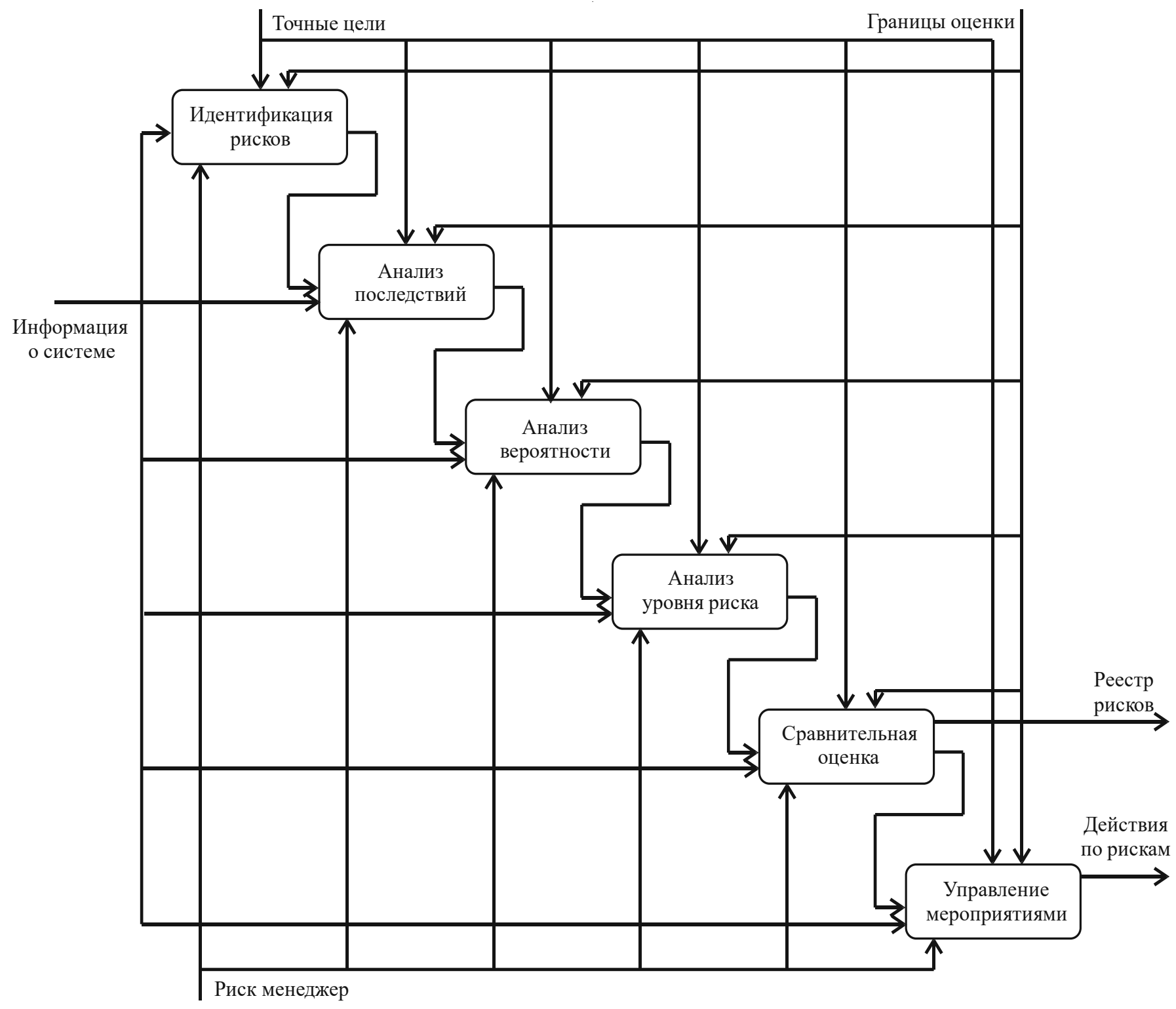

Рис. 1. Общий процесс управления рисками в виде модели IDEF0

Согласно стандарту ГОСТ Р ИСО 9001:2015 [1], который включает себя требования относительно управления рисками, процессный подход позволяет:

а) понимать и постоянно выполнять требования;

b) рассматривать процессы с точки зрения добавления ими ценности;

с) достигать результативного функционирования процессов;

d) улучшать процессы на основе оценивания данных и информации.

Использование процессного подхода при комбинировании технологий оценки рисков предполагает формализованное описание границ действия технологий и результатов процессов идентификации рисков, анализа рисков и сравнительной оценки рисков.

Для уменьшения «порога вхождения» в процессы оценки рисков и применения процессного подхода оптимальным является набор графических моделей в широко используемой нотации, поддерживающей возможность выделения границ процессов и передачи между ними объектов. Такой нотацией является IDEF0.

Опишем общий процесс управления рисками в виде модели IDEF0 (рисунок 1).

Процесс управления рисками предполагает идентификацию рисков, а именно выявление в заданных гра- 


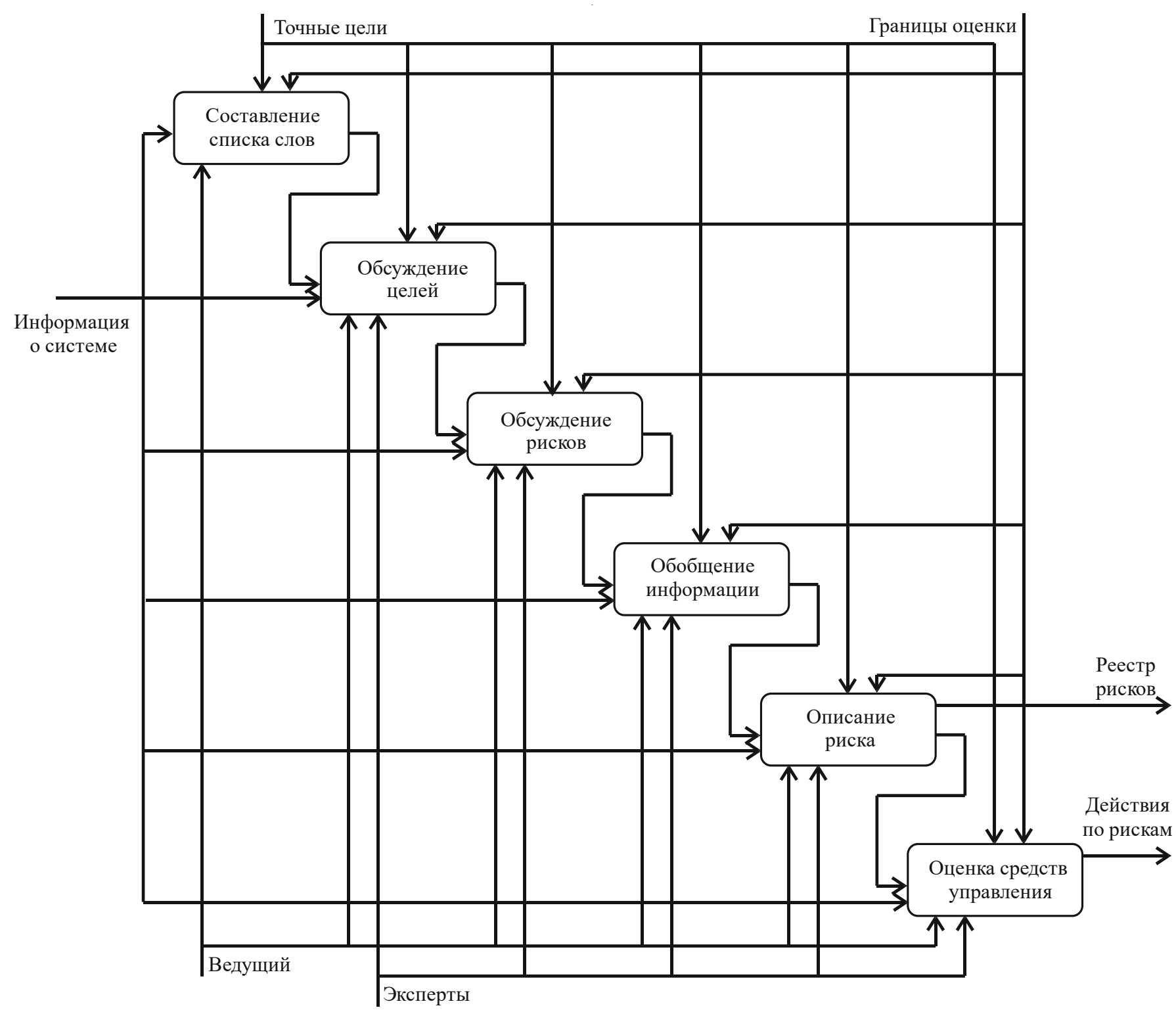

Рис. 2. Технология оценки рисков «Структурированный анализ сценариев методом «что, если?» (SWIFT)» в виде модели IDEF0

ницах (начальных и граничных условиях) максимального числа рисков. На следующем этапе проводится анализ последствий и вероятности их возникновения. Также может быть проанализирована вероятность необнаружения риска. На основе полученных данных рассчитывается уровень риска и проводится сравнительная оценка (ранжирование) всех рисков. Завершающим этапом является выработка предложений и исполнение мероприятий, направленных на обработку рисков.

Входными данными для описанного процесса являются информация о системе и объекте оценки, точные цели и границы оценки. Итогом процесса оценки ри- сков является разработанный реестр рисков, включающий в себя информацию по рискам и их показателям, и предложения по мероприятиям в отношении рисков.

Технологии оценки рисков [2] применимы на разных этапах процесса управления рисками: в рамках идентификации рисков, анализа рисков либо сравнительной оценки рисков - и имеются определённые входы и выходы. Из этого следует, что использование формализованного процессного подхода позволяет проводить комбинирование технологий, с использованием универсальных «типов» выходов и входов. Так результатом идентификации рисков для всех технологий является набор рисков. Результатом анализа являются 


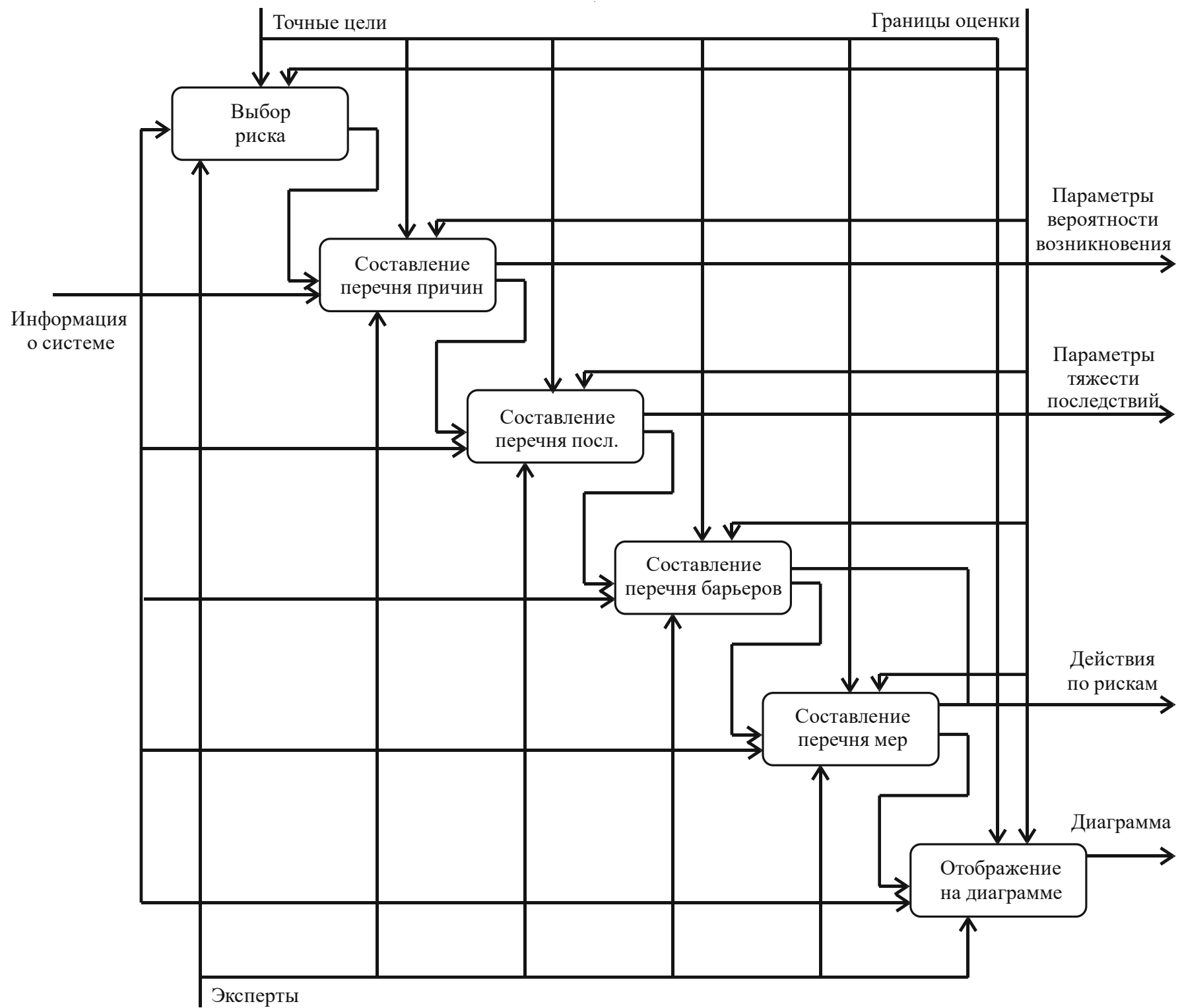

Рис. 3. Технология оценки рисков «Анализ «галстук-бабочка» в виде модели IDEF0

параметры их вероятности и последствий (и вероятности необнаружения). Сравнительная оценка завершается выставленными приоритетами работы по рискам. Особенности технологий могут быть представлены дочерними процессами каждого из этапов оценки рисков.

Рассмотрим вариант комбинирования технологий с применением процессного подхода на примере двух технологий: SWIFT и диаграммы галстук-бабочка. Так на рис. 2 представлена технология оценки рисков «Структурированный анализ сценариев методом «что, если?» (SWIFT)» в виде модели IDEFO.

Первые четыре этапа относятся к идентификации рисков и их результатом является набор рисков. Пятый этап относится к анализу рисков и их результат представлен параметрами вероятности и последствий (и вероятности необнаружения) рисков. Шестой к управлению мероприятиями по рискам с результирующим набором мероприятий по рискам. Такое точное разделение процессов позволяет применить технологию, к примеру, только для идентификации рисков.

На рисунке 3 представлена технология оценки рисков «Анализ «галстук-бабочка» в виде модели IDEFO.

Первые три этапа относятся к анализу рисков и их результат представлен параметрами вероятности и последствий рисков. Четвёртый и пятый к управлению мероприятиями по рискам с результирующим набором 
мероприятий по рискам. Такое точное разделение процессов позволяет применить технологию, к примеру, только для анализа рисков.

Используя соответствующие этапы технологий «Структурированный анализ сценариев методом «что, если?» (SWIFT)» и «Анализ «галстук-бабочка» для идентификации и анализа рисков соответственно, можно получить комбинированную технологию оценки рисков, включающую в себя достоинства обоих технологий.

Сочетание технологий и их представление в виде сети взаимосвязанных процессов, применяемое в рамках управления рисками, позволяет получить множество комбинаций технологий оценки рисков, что в свою очередь даёт возможность более точного выбора подходящей комбинации, учитывающих особенности вида деятельности или параметров организации. Формализованность процессов по входам и выходам подразумевает их взаимозаменяемость с точки зрения процедур. Идентификацию рисков можно проводить одной технологией, а анализ рисков другим. При этом на каждый риск можно выбрать свою технологию анализа, при использовании одинаковой технологии идентификации, т.к. идентификации проводится для всех рисков, а анализ для одного конкретного риска. Учитывая этот факт, возможно применение различных сгруппированных подходов комбинирования [5]:
1. последовательное комбинирование (идентификация - технология А; анализ - технология В);

2. параллельное комбинирование (идентификация - технология A, технология B; анализ - технология $\mathrm{C}$, технология $\mathrm{D}$, технология $\mathrm{E})$;

3. последовательно-параллельное комбинирование (идентификация - технология $A$; анализ технология $\mathrm{B}$, технология $\mathrm{C}$, технология $\mathrm{D})$;

4. параллельно-последовательное комбинирование (идентификация - технология A, технология B; анализ - технология C).

У каждого способа комбинирования имеются свои преимущества и недостатки, связанные с точностью оценки и затрачиваемыми временными и квалификационными ресурсами. Так, к примеру, использование последовательно-параллельного комбинирования при условии распараллеливания технологий анализа рисков по рискам (на каждый риск своя технология анализа рисков) позволяет получить больший эффективный результат при относительно малых требованиях к подготовке (достаточно разделения процедур по этапам) технологии. Данный подход был использован при разработке комплекса программных средств для оценки рисков KuroT Risks (свидетельство о регистрации программы для ЭВМ № 2017618827), апробация которого продемонстрировала увеличение эффективности анализа причин возникновения брака при производстве в 4,5 раза по сравнению с традиционным выполнением процесса.

\section{ЛИТЕРАТУРА}

1. ГОСТ Р ИСО 9001:2015. Системы менеджмента качества. Требования.- М.: Стандартинформ, 2020.— 32 с.

2. ГоСТ Р 58771:2019. Менеджмент риска. Технологии оценки риска.- М.: Стандартинформ, 2020. - 90 c.

3. Tikhonov M.R. Algorithmic support for risk assessment in electronic production management / Tikhonov M.R., Slyusar V.V., Akulenok M.V., Andrianov A.M., Slyusar M. // 2021 IEEE Conference of Russian Young Researchers in Electrical and Electronic Engineering (ElConRus), 2021, pp. 2262-2265

4. Тихонов М.Р. Сравнительный анализ автоматизированных систем управления рисками / Тихонов М.Р., Акуленок М.В.// Автоматизация. Современные Технологии. — № 9 2019. Москва 2019.— - . 387-391.

5. Тихонов М.Р. Классификация способов комбинирования методов оценки рисков // Современная наука: актуальные проблемы теории и практики. Серия «Естественные и технические науки».—№ 9, —2018. — с. 64-69.

6. Тихонов М.Р. Риск-ориентированный анализ процесса информационного обеспечения предприятия / Тихонов М.Р., Акуленок М.В., Сударикова А.А. // 0боронный комплекс — научно-техническому прогрессу России — № 4 2016. Москва 2016. 3-8с.

7. Adam S. Markowski. "Bow-tie" model in layer of protection analysis / Adam S. Markowski, Agata Kotynia // Process Safety and Environmental Protection. 2011. — № 89. - P. 205-213

(c) Тихонов Мартин Робертович (kurotenshi91@yandex.ru),

Акуленок Марина Викторовна ( amv@s2q.ru ), Шикула Ольга Сергеевна ( oshik78@mail.ru ).

Журнал «Современная наука: актуальные проблемы теории и практики» 